\title{
A broad-spectrum antibiotic, DCAP, reduces uropathogenic Escherichia coli infection and enhances vorinostat anticancer activity by modulating autophagy
}

\author{
Giulia Allavena', Doriana Debellis², Roberto Marotta ${ }^{2}$, Chetanchandra S. Joshi ${ }^{3}$, Indira U. Mysorekar ${ }^{3,4}$ and \\ Benedetto Grimaldi (i)
}

\begin{abstract}
The cellular recycling pathway of autophagy plays a fundamental role in adaptive responses to nutrient deprivation and other forms of stress under physiological and pathological conditions. However, autophagy can also be a doubleedge sword during certain bacterial infections (such as urinary tract infections) and in cancer, where it can be hijacked by the pathogens and cancer cells, respectively, to promote their own survival. Thus, autophagy modulation can potentially have multiple effects in multiple contexts and this property can be leveraged to improve outcomes. In this report, we identify that a broad-spectrum antibiotic, 2-((3-(3, 6-dichloro-9H-carbazol-9-yl)-2-hydroxypropyl) amino)-2(hydroxymethyl) propane-1, 3-diol (DCAP) modulates autophagy. We employed combined biochemical, fluorescence microscopy and correlative light electron microscopy approaches to demonstrate that DCAP treatment blocks autophagy at the late stages by preventing autophagolysosome maturation and interrupting the autophagic flux. We further show that, DCAP significantly reduces UPEC infection in urinary tract epithelial cells via inhibition of autophagy. Finally, we reveal that DCAP enhances the anticancer activity of the histone acetyltransferase (HDAC) inhibitor, vorinostat, which has been reported to increase susceptibility to bacterial infections as a common adverse effect. Collectively, our data support the concept that DCAP represents a valuable chemical scaffold for the development of an innovative class of bactericidal autophagy inhibitors for treatment of urinary tract infections and/or for adjuvant therapy in cancer treatment.
\end{abstract}

\section{Introduction}

Macroautophagy (henceforth referred to as autophagy) is a proteosomal-independent degradative mechanism that promotes catabolism and recycling of diverse

\footnotetext{
Correspondence: Benedetto Grimaldi (benedetto.grimaldi@iit.it)

'Laboratory of Molecular Medicine, Fondazione Istituto Italiano di Tecnologia, via Morego 30, 16163 Genova, Italy

2Electron Microscopy facility, Fondazione Istituto Italiano di Tecnologia, via

Morego 30, 16163 Genova, Italy

Full list of author information is available at the end of the article.

Edited by P. Salomoni
}

cytoplasmic content ${ }^{1,2}$. A number of autophagy-related genes (ATGs) are implicated in the formation and maturation of cytoplasmic double membrane vesicles, named autophagosomes, which engulf a variety of macromolecules and organelles. Mature autophagosomes fuse with lysosomes to form digestive acidic vescicles, called autophagolysosomes or autolysosomes and contents are degraded and recycled ${ }^{3-5}$.

A key autophagy gene/protein component is the microtubule Associated Protein 1 Light Chain 3 (LC3),

\section{(c) The Author(s) 2018}

(c) (i) Open Access This article is licensed under a Creative Commons Attribution 4.0 International License, which permits use, sharing, adaptation, distribution and reproduction c. in any medium or format, as long as you give appropriate credit to the original author(s) and the source, provide a link to the Creative Commons license, and indicate if changes were made. The images or other third party material in this article are included in the article's Creative Commons license, unless indicated otherwise in a credit line to the material. If material is not included in the article's Creative Commons license and your intended use is not permitted by statutory regulation or exceeds the permitted use, you will need to obtain permission directly from the copyright holder. To view a copy of this license, visit http://creativecommons.org/licenses/by/4.0/. 

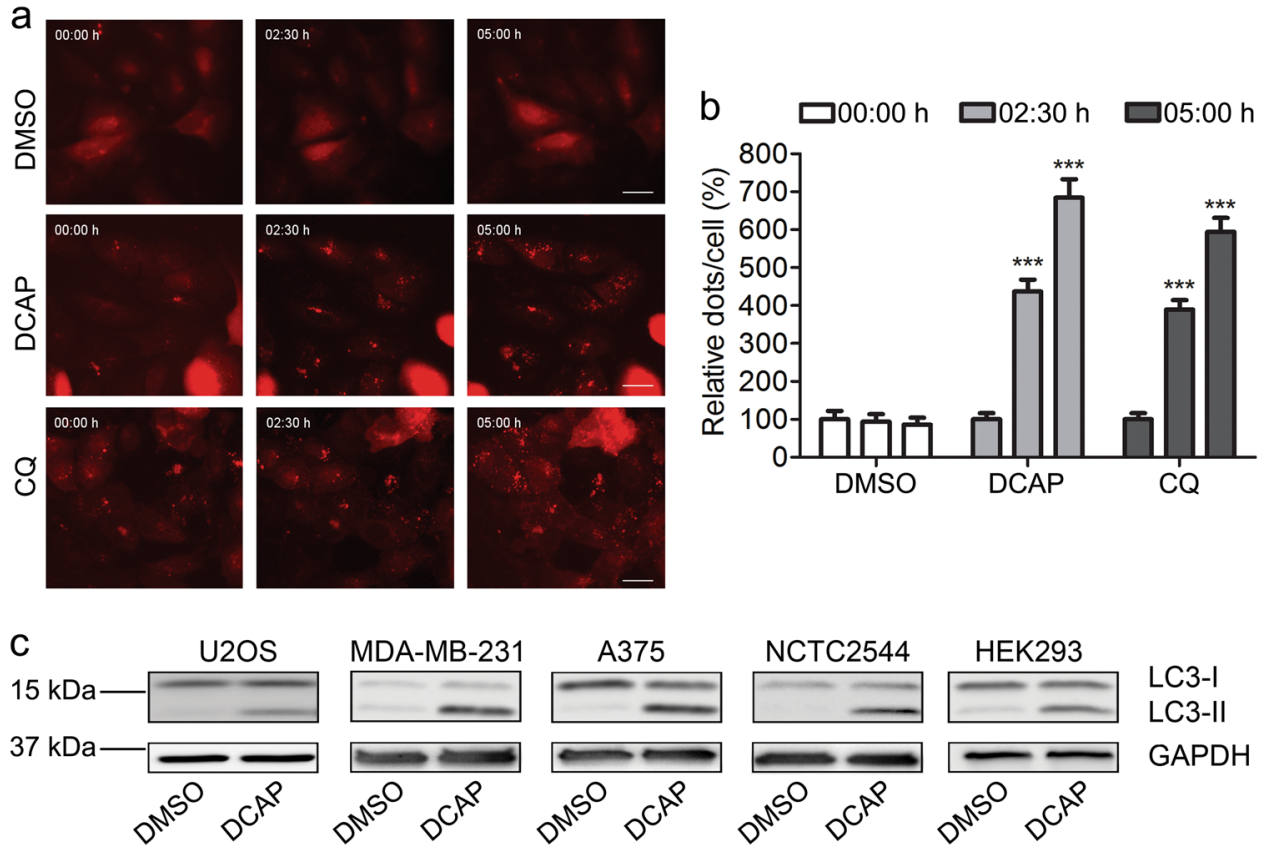

LC3-I

LC3-II

GAPDH

Fig. 1 A broad-spectrum antibiotic, DCAP, modulates autophagy in human cells. a Human U2OS cells expressing the autophagic marker, LC3, fused with a red fluorescent protein (LC3-RFP) were treated with DMSO and equimolar concentration (50 $\mu \mathrm{M})$ of the autophagy inhibitor, chloroquine (CQ), or the antibiotic, DCAP. Series of image frames of LC3-RFP were acquired by fluorescence microscopy at $25 \mathrm{~min} /$ frame over a $12 \mathrm{~h}$ period (Supplementary Video S1, S2 and S3). Representative sequences of images at 0, 2.5 and $5 \mathrm{~h}$ displaying accumulation of LC3-RFP dots overtime in DCAP and CQ-treated cells are shown. $\mathbf{b}$ Quantification of the number of fluorescent LC3-RFP dots per cell after a 2.5 and $5 \mathrm{~h}$ treatment with DMSO, DCAP or CQ. The number of fluorescent dots at time zero was set to $100 \%$. Shown as mean percentage of relative dots/cell \pm s.e.m., ${ }^{* * * P<}$ 0.001, compounds versus DMSO (two-way ANOVA with Bonferroni post tests). c Immunoblot analysis of unconjugated (LC3-I) and lipid-conjugated (LC3-II) form of the autophagy marker, LC3, across human bone (U2OS), mammary gland (MDA-MB-231), skin (A375, NCTC 2544) and embryonic kidney (HEK 293) cultured cells treated $24 \mathrm{~h}$ with vehicle (DMSO) or $10 \mu \mathrm{M}$ DCAP. GADPH was used as a loading control

lipid-conjugated LC3 is specifically recruited to the autophagosomal membranes, assisting both formation and maturation of autophagosomes ${ }^{3}$. Accordingly, the evaluation of lipid-modified LC3 and/or its cellular localization serves as a suitable system to monitor autophagosome turnover under diverse conditions ${ }^{6,7}$. In addition to ATGs, scaffolding/adaptor proteins like p62 also known as sequestosome 1 (SQSTM1) assist the recruitment of specific targets to the autophagomes. p62/SQSTM1, serves as a link between LC3 and ubiquitinated substrates ${ }^{8}$. SQSTM1 and SQSTM1bound polyubiquitinated proteins are incorporated into the completed autophagosome and are degraded in autolysosomes ${ }^{9}$.

Accumulating evidence indicates that autophagy is a fundamental adaptive response to starvation and other forms of stress ${ }^{10-12}$, in tissue homeostasis ${ }^{13}$, cellular differentiation and development ${ }^{14}$, and ageing ${ }^{15,16}$. Thus, pharmacological induction of autophagy has been proposed for preventing the development of human pathologies or for reversing the adverse effects of ageing ${ }^{17}$.

Nonetheless, autophagy can also be detrimental as is the case with certain pathogens that co-opt the pathway for their survival. For example, uropathogenic Escherichia coli (UPEC), the predominant cause of Urinary Tract Infections (UTIs), can persist within the urinary bladder epithelium (urothelium) by hijacking the autophagy pathway, forming quiescent intracellular reservoirs within autophagosomes wherein they are refractory to antibiotic treatment ${ }^{18,19}$.

On the other hand, in case of cancer, established tumor cells often enhance autophagic flux to increase their survival under limited nutrition conditions and/or to overcome chemotherapy induced stress by recycling cellular components to produce alternative sources of energy. ${ }^{20-22}$. Consequently, a block of autophagy has been proposed as a suitable strategy for improving the anticancer activity of several neoplastic agents ${ }^{21}$. As an example, genetic or pharmacological inhibition of autophagy in breast cancer cells improved the anticancer activity of the histone de-acetyltransferase (HDAC) inhibitor, vorinostat ${ }^{7,23}$.

Here we show that a recently identified broad-spectrum antibiotic, DCAP (2-((3-(3, 6-dichloro-9H-carbazol-9-yl)2-hydroxypropyl) amino)-2-(hydroxymethyl) propane-1, 3 -diol), can inhibit canonical autophagy in human cancer 
cells by blocking autophagic flux and preventing maturation of autophagolysosomes. Further, DCAPmediated autophagy blockade limits UPEC infection of bladder cells. Finally, DCAP enhances the anticancer activity of vorinostat against breast cancer cells. Thus, we propose DCAP as a valuable chemical scaffold for the

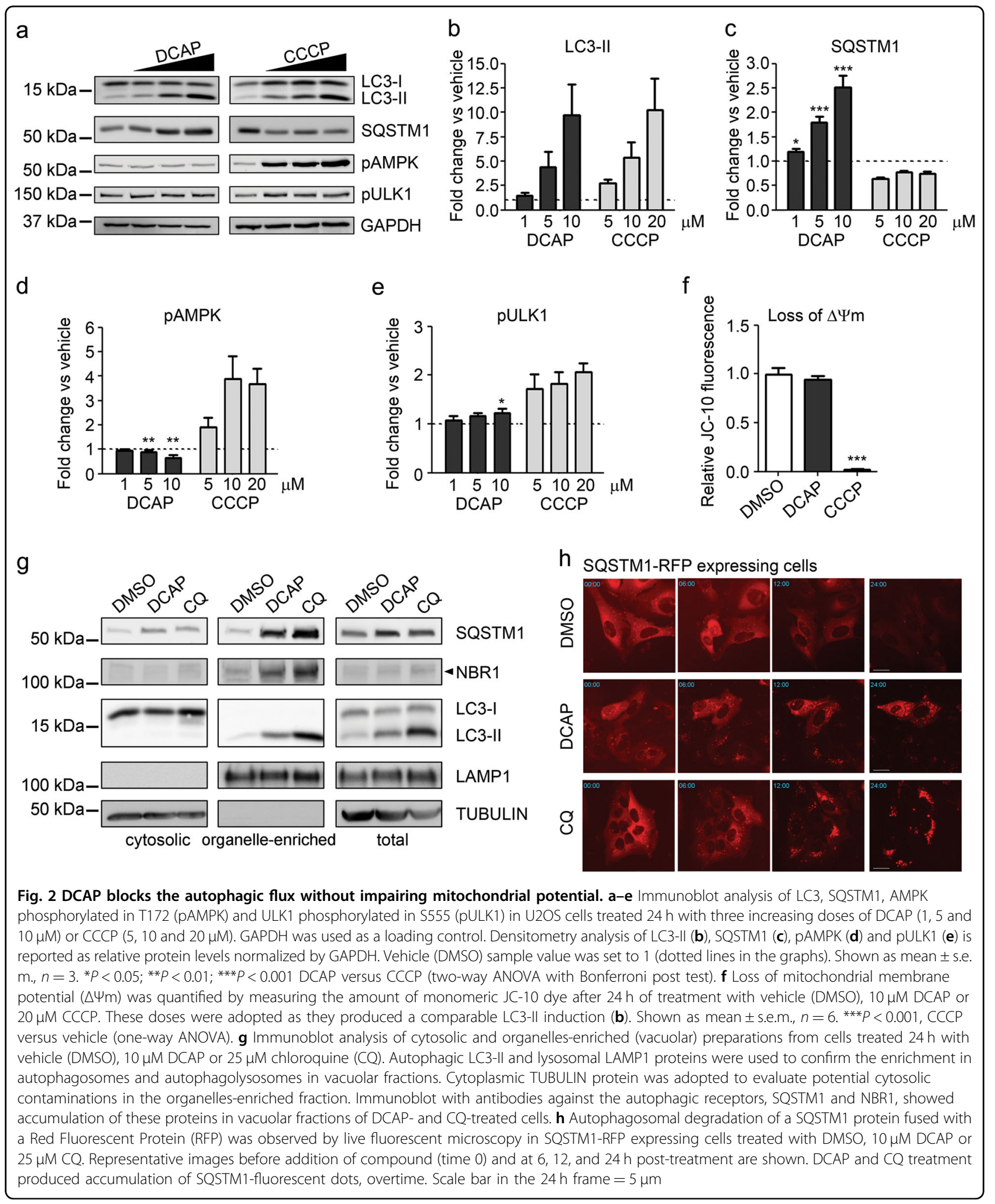


development of antibacterial, anti-autophagic drugs to treat UTIs and for use as combination therapy agents along with vorinostat in breast cancer treatment.

\section{Results \\ DCAP, a broad-spectrum antibiotic, negatively modulates autophagy and inhibits autophagic degradation}

To identify novel small molecule compounds affecting autophagy, human osteosarcoma U2OS cells expressing the protein LC3 (a marker of autophagosomes) fused with a Red Fluorescent Protein (RFP-LC3) were monitored by real-time fluorescence imaging in presence or absence of selected compounds. A well-defined autophagy inhibitor, chloroquine (CQ), was adopted as a reference. U2OS RFP-LC3 cells treated with CQ showed a significant accumulation of LC3-RFP-fluorescent dots overtime, compared with vehicle (DMSO) (Fig. 1a, b and Supplementary Video S1 and S2).

With this asset, we screened a subset of small molecules present in the internal chemical collection of the Ististuto Italiano di Tecnologia (Italy), which contains both commercially available and in-house synthetized compounds. Among them, we selected $\sim 200$ diverse and nonredundant molecules with structural and chemical features different from current characterized autophagy modulators, including polyamines ${ }^{24}$, quinolones ${ }^{25}$ and cyclic-substituted amines ${ }^{7,26}$ ). This screening revealed that a carbazol-containing compound previously characterized as a broad-spectrum antibiotic, 2-((3- $(3,6-$ dichloro-9H-carbazol-9-yl)-2-hydroxypropyl) amino)-2(hydroxymethyl) propane-1, 3-diol (DCAP) was a novel autophagy modulator. Indeed, DCAP stimulated the formation of RPF-LC3 puncta when added to U2OS cells (Fig. 1a, b and Supplementary Video S3).

Validating our fluorescence microscopy analysis, and extending our observation to additional human cell lines, we found that $10 \mu \mathrm{M}$ DCAP increased the levels of endogenous lipidated LC3 (LC3-II) in cell lines from bone (U2OS), mammary gland (MDA-MB-231), skin (A375 and NCTC2544) and kidney (HEK-293) (Fig. 1c). Indicating a specific activity of DCAP toward autophagy, two additional commercially available carbazol-containing compounds were not active in our screening and did not significantly increased LC3-II protein levels in U2OS cells (Supplementary Fig. S1).

DCAP was previously shown to disrupt bacterial membranes by reducing transmembrane potential of Gram-positive and Gram-negative bacteria ${ }^{27}$. However, high doses of DCAP also affected mitochondrial membrane potential of human cells, similar to the ionophore carbonyl cyanide m-chlorophenyl hydrazone (CCCP), whose AMPK-dependent autophagy-inducing activity is well characterized $^{27,28}$. We thus compared the effects of DCAP and CCCP on the levels of lipid-conjugated LC3
(LC3-II), SQSTM1, and phosphorylated AMPK proteins by immunoblot analysis with specific antibodies (Fig. 2a). DCAP- and CCCP-treated cells accumulated high levels of LC3-II, thus confirming that both compounds affected autophagy (Fig. 2b). However, the two compounds generated significant opposing effects on SQSTM1 levels (Fig. 2c). While CCCP enhanced SQSTM1 autophagymediated degradation, thus reducing SQSTM1 signal, DCAP produced a significant accumulation of SQSTM1 in a dose-dependent manner. Furthermore, DCAP produced negligible differences in the levels of phosphorylated AMPK and its autophagy-related target, ULK1, compared with CCCP, which enhanced the signals of both phosphorylated proteins (Fig. 2d, e).

A lack of AMPK activation by DCAP indicates that this compound does not affect autophagy by impairing mitochondrial potential $(\Delta \Psi \mathrm{m})$ at the doses tested. To validate this, we assessed the $\triangle \Psi \mathrm{m}$ in U2OS cells treated with a dose of DCAP and CCCP that produced a comparable increase in LC3-II levels (10 and $20 \mu \mathrm{M}$, respectivelyFig. 2b). Unlike CCCP, which induced a drastic loss of $\triangle \Psi \mathrm{m}$, DCAP was completely ineffective (Fig. 2f).

Our observations suggest that DCAP may block autophagy-mediated protein degradation, thus accumulating immature autophagosomes and/or autophagolysosomes. To validate this hypothesis, we prepared cytosolic and organelle-enriched fractions from cells treated with DCAP or vehicle. Because CQ enhances cellular autophagolysosome formation ${ }^{7}$, this compound was adopted as a reference. Immunoblot analysis with antibodies against LC3 confirmed the enrichment of autophagosomes/ autophagolysosomes in the organelle fractions, which predominantly contained the membrane-bound LC3-II form (Fig. 2g). Consistent with a DCAP-mediated blockade of the autophagic flux, cells treated with this compound showed a marked accumulation of both LC3-II and SQSTM1 in organelle-enriched fractions, compared with vehicle (Fig. 2g). Furthermore, the vacuolar levels of an additional autophagic receptor, $\mathrm{NBR} 1^{29}$, were also increased by the treatment with DCAP and CQ (Fig. 2g).

We further analyzed the levels of lipidated LC3-II in cells treated with DMSO, DCAP or CCCP in presence with the late-stage autophagy inhibitor, Bafilomycin A1 (Supplementary Fig. S2). As expected by a treatment with an autophagy inducer ${ }^{30}$, a co-treatment with CCCP and Bafilomycin A1 significantly increased the levels of LC3-II compared with DMSO and Bafilomycin A1 (Supplementary Fig. S2b). In marked contrast, the combination of DCAP and Bafilomycin A1 generated negligible differences compared with DMSO and Bafilomycin A1 treatment (Supplementary Fig. S2b). A lack of DCAP-mediated accumulation of membrane-bound LC3-II in presence of Bafilomycin A1 was also confirmed in organelle-enriched preparations (Supplementary Fig. S2c). 
As an independent experimental validation that DCAP blocks the autophagic flux, we monitored the autophagymediated degradation of a SQSTM1-RFP fusion protein by time-course fluorescent microscopy. The addition of DCAP to the medium resulted in a progressive accumulation of SQSTM1-RFP punctae (Fig. 2h and Supplementary Video S4), confirming that DCAP inhibits autophagy-dependent SQSTM1 turnover.

Collectively, our data demonstrate that DCAP possesses autophagic inhibitory activity and blocks autophagic degradation.

\section{DCAP blocks autophagy at the last stage by impairing autophagolysosome degradation}

The fact that DCAP-treated cells showed accumulation of autophagic receptors in organelle-enriched fractions (Fig. 2g) implies that this antibiotic blocks the late stage of autophagy by either preventing autophagosome/lysosome fusion or avoiding autophagolysosome maturation. To discriminate between these two diverse mechanisms, we conducted Correlative Light Electron Microscopy (CLEM) analysis on SQSTM1-RFP-expressing cells, which was grown on gridded coverslip and treated with $10 \mu \mathrm{M}$ of DCAP for $24 \mathrm{~h}$. After nuclear staining with Hoechst-33342, fluorescence images were acquired and confirmed the presence of numerous perinuclear SQSTM1-RFP dots. The samples imaged at the fluorescence microscope were next processed for TEM, obtaining ultrastructural information of the same cells analyzed by fluorescent microscopy (see representative cells in Fig. 3a, d). The CLEM analysis showed cytoplasmic accumulation of late degradative vacuoles in correspondence of the regions containing SQSTM1-fluorescent dots, characterized by a partially or completely absence of internal membranes and by the presence of degraded cytoplasmic material that appear strongly electron dense (dark) in osmium tetroxide post-fixed TEM sections ${ }^{9}$ (Fig. 3c, f).

The morphological feature of the observed vacuoles, together with the presence of SQSTM1 fluorescence, clearly identify these structures as late autophagolysosomes and indicates that DCAP inhibits autophagy at its last step.

In line with this concept, quantitative TEM analysis showed significant increase of degradative vacuoles in DCAP-treated cells, compared with DMSO treated cells (Fig. 3g, h).

\section{DCAP prevents lysosomal acidification without affecting lysosomal membrane permeabilization}

The observed accumulation of autophagolysosomal structures in DCAP-treated cells indicates that this antibiotic may impair lysosomal function. We thus assessed whether DCAP may affect lysosomal membrane stability and induce lysosomal membrane permeabilization $(\mathrm{LMP})^{31}$. Several soluble carbohydrate-binding lectins, such as Galectin 1, translocate to the sites of endolysosomal leakage upon LMP and the evaluation of galectin puncta formation by immunofluorescence microscopy has been proven to be a specific method for LMP assessment ${ }^{32}$. Accordingly, we evaluated the number of Galectin 1 puncta in cells treated with DCAP or with Lleucyl-L-leucine methyl ester (LLOMe), which specifically induces lysosome membrane damage ${ }^{32}$. In addition, we monitored the autophagic flux by assessing the amount of SQSTM1 fluorescent dots. As expected, LLOMe-treated cells accumulated numerous Galectin 1-positive fluorescence dots (Fig. 4a, b). In marked contrast, DCAP did not significantly affected the number of Galectin 1 puncta, while it generated a significant accumulation of SQSTM1positive dots (Fig. $4 \mathrm{a}-\mathrm{c}$ ).

Considering the LMP-independent autophagolysosome inhibition activity of DCAP, we determined whether this compound might block autophagy by affecting lysosomal $\mathrm{pH}$, which is the mechanism of action of several known autophagy inhibitors, including $\mathrm{CQ}^{33}$. To this aim, we compared the ability of DCAP or CQ in affecting the ratio between the blue basic and green acidic form of a fluorescent $\mathrm{pH}$ indicator. The treatment with DCAP or CQ generated similar and significant increase in basic/acidic ratio of the indicator as compared with vehicle, thus showing inhibition of lysosomal acidification (Fig. 4d, e).

Thus, our data demonstrate that DCAP blocks the late stage of autophagy by inhibiting lysosomal acidification, hence preventing the final maturation/degradation step of autophagolysosomes.

\section{DCAP reduces autophagy-mediated UPEC infection}

Considering the role of autophagy in the pathogenesis of UTIs ${ }^{18,19}$, wherein UPEC hijack the pathway to persist in autophagosomes, the fact that a broad-spectrum antibiotic affected autophagy warranted further investigation. We inquired this possibility in an in vitro infection assay in 5637 bladder epithelial cells (BECs) infected with a pathogenic E. coli strain UTI89 ${ }^{10}$. We first determined the cytotoxicity of DCAP against UTI89 cells. A doseresponse treatment of free bacteria with diverse doses of DCAP revealed that this antibiotic was effective in reducing bacterial growth starting from $30 \mu \mathrm{M}$ (Fig. 5a). Further analysis showed that the minimal inhibitory concentration (MIC) of DCAP against UTI89 pathogenic bacteria was in the low/medium micromolar range, thus comparable with the MIC of a well-characterized betalactam antibiotic, ampicillin (Fig. 5b). In marked contrast, the single autophagy inhibitor, CQ, was completely ineffective against bacteria at a dose as high as $4 \mathrm{mM}$ (Fig. 5b).

Considering that $10 \mu \mathrm{M}$ DCAP did not affected the growth of free UPEC bacteria (Fig. 5a), we decided to 

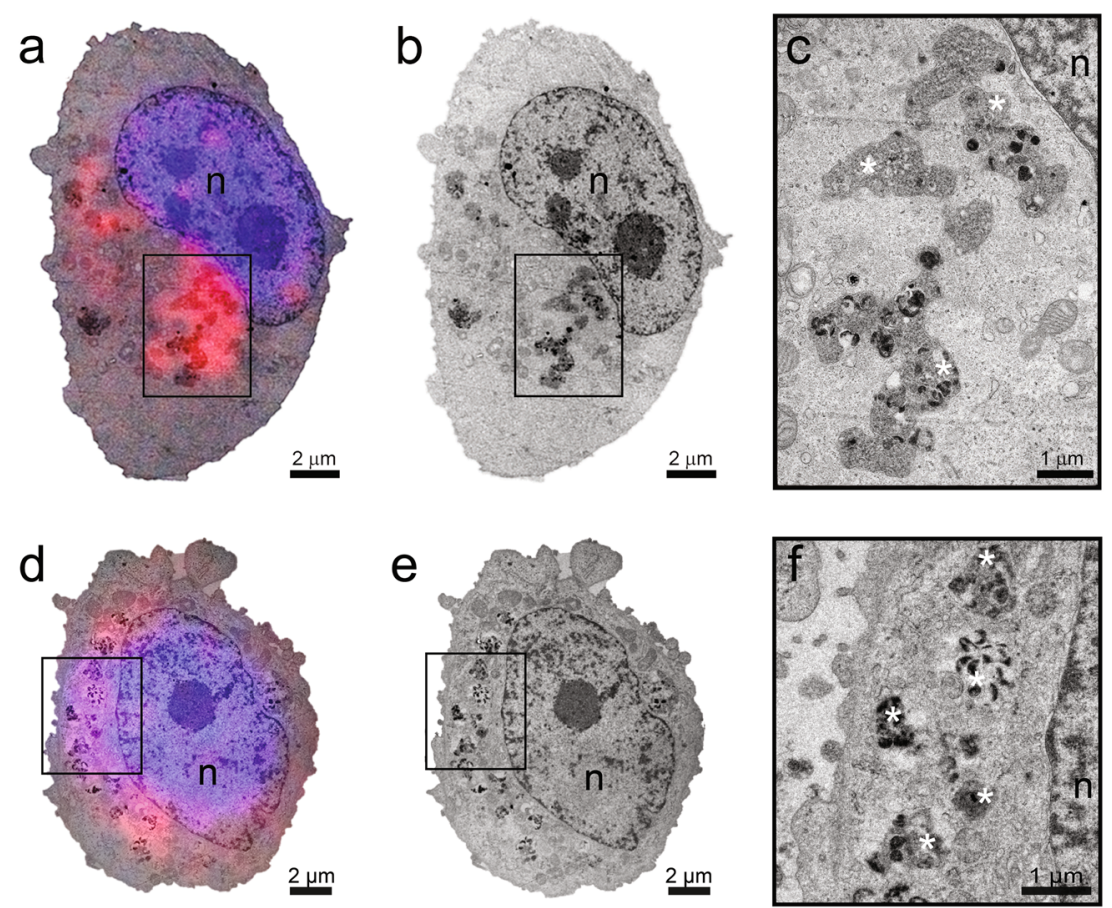

g

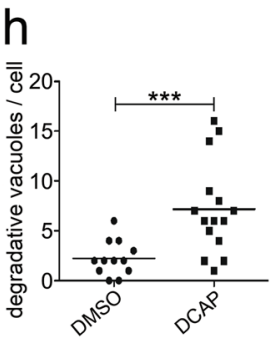

Fig. 3 DCAP blocks autophagy at the late stage. Correlative light electron microscopy (CLEM) on SQSTM1-RFP expressing cells treated with $10 \mu M$ of DCAP for $24 \mathrm{~h}$. a, $\mathbf{d}$ Superimposed maximal projections of $\mathrm{z}$-stack confocal images and low magnification TEM slice projection image of two entire representative DCAP-treated cells. b, e TEM projection image of the same cells shown in $(\mathbf{a}, \mathbf{d})$. c, $\mathbf{f}$ High magnification of the region boxed in (a, $\mathbf{d})$ showing a cluster of SQSTM1-fluorescent positive degradative autophagic vacuoles (AVd) containing electron dense material (dark) and membrane remnants (asterisks). $\mathbf{g}$, $\mathbf{h}$ Quantitative assessment of degradative vacuoles in TEM sections from cells treated with DMSO or $10 \mu M$ DCAP. Example of cytoplasmic regions containing degradative vacuoles (asterisks) in DCAP-treated section is provided in $\mathbf{g}$. Quantification in (h) is shown as mean degradative vacuoles per cell \pm s.e.m., ${ }^{* * *} P<0.001$ DCAP versus DMSO (Mann-Whitney test)

adopt this dose to specifically test a potential bactericidalindependent/autophagy-dependent activity of DCAP against intracellular bacteria. Similar to the observation in other human cell lines (Supplementary Fig. S3), $10 \mu \mathrm{M}$ of DCAP markedly affected autophagy, but not viability, of BECs (Fig. 5c, d). Yet, this dose significantly reduced the number of intracellular bacteria in UPEC-infected BECs (Fig. 5e).

To verify that DCAP-mediated reduction of intracellular infection actually derived from a DCAP-dependent blockade of autophagy, we repeated the UPEC infection in BECs in which the expression of essential autophagy protein, ATG16L1, was silenced by siRNA (Fig. 5f). While scrambled control siRNA treated with DCAP showed a significant decrease in bacterial CFUs, the treatment of
ATG16L1 silenced cells did not alter bacterial titers (Fig. 5g). Collectively, these data indicate that DCAP displays autophagy-mediated antibacterial activity.

\section{DCAP improves the in vitro anticancer activity of the HDAC inhibitor, vorinostat}

In vitro studies indicated that autophagy plays a protective role in vorinostat-induced cytotoxicity against cancer cells ${ }^{23}$. We thus evaluated the ability of DCAP in improving the anticancer activity of the HDAC inhibitor (HDACi), vorinostat, against breast cancer MDA-MB-231 cells. In presence of a sub-lethal dose of DCAP, vorinostat in vitro anticancer activity significantly improved and the concentration of this HDACi required to kill $50 \%$ of cells $\left(\mathrm{IC}_{50}\right)$ decreased by a logarithmic factor (Fig. 6a). 


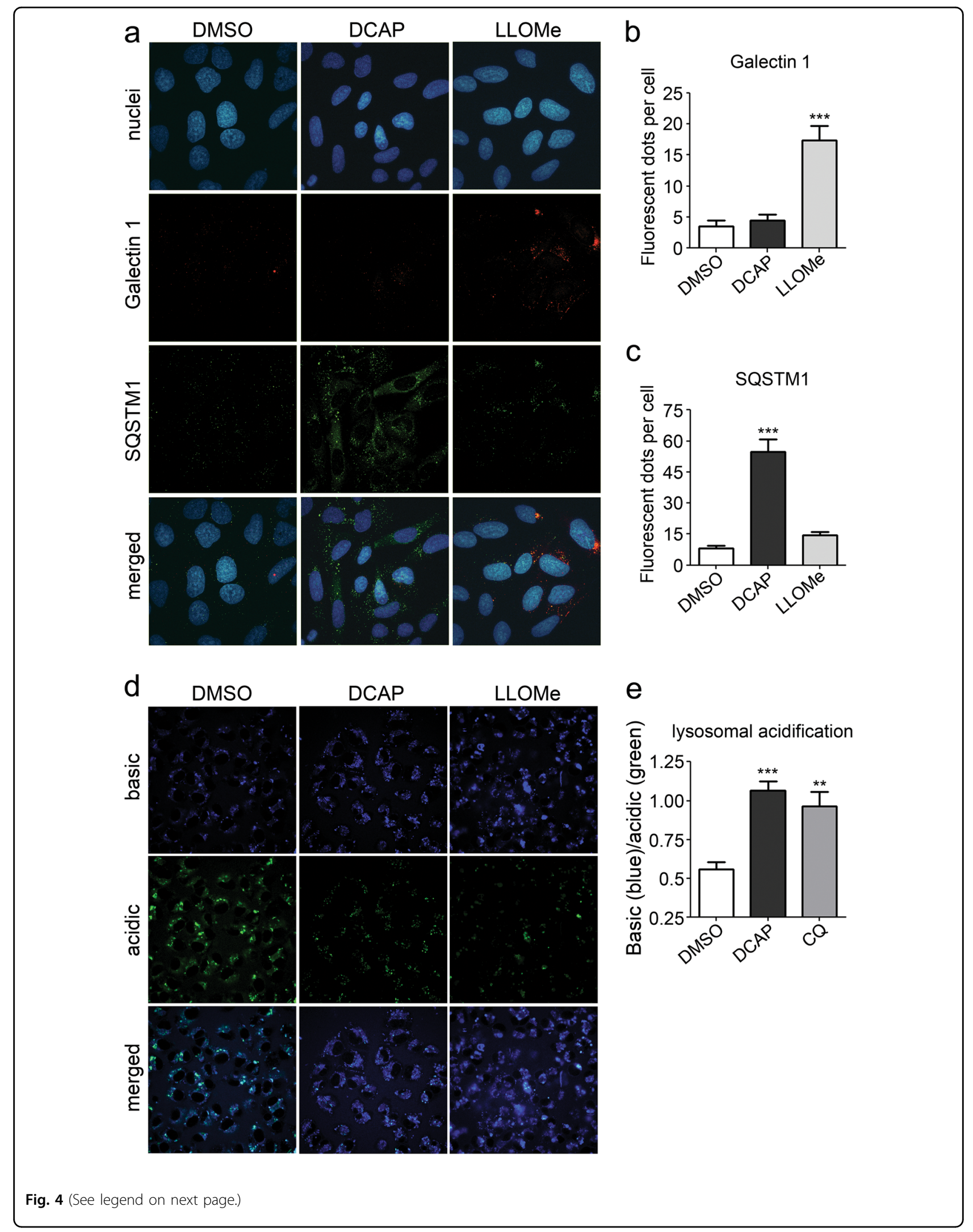


(see figure on previous page)

Fig. 4 DCAP inhibits lysosome acidification without affecting lysosomal membrane permeability. a-c Lysosomal membrane permeabilization (LMP) and autophagy in cells treated with vehicle, $10 \mu \mathrm{M}$ DCAP or L-leucyl-L-leucine methyl ester (LLOMe), which specifically induces lysosome membrane damage, was assessed by immunofluorescence microscopy with antibodies against a LMP-related marker, Galectin 1, and the autophagic receptor, SQSTM1. a Representative confocal images of treated cells probed with an anti-Galectin 1/ antibody/Alexa555 (red), anti-SQSTM1/Alexa488 (green) and Hoechst 33342 nuclear staining (blue). b, c Quantification of Galectin 1- and SQSTM1-positive fluorescent dots per cell. Shown as average number of fluorescent dots per cell \pm s.e.m., $n=3$. ${ }^{* * *} P<0.001$, compounds vs. DMSO (one-way ANOVA, Dunnett's Multiple Comparison Test). d, e The effect of $10 \mu \mathrm{M}$ DCAP or $25 \mu \mathrm{M} C Q$ in decreasing lysosomal acidification was assessed by measuring the ratio between blue (basic) and green (acidic) fluorescent signals of a lysosomal pH indicator, LysoSensor Yellow/Blue dextran. Representative fluorescent images are shown in $\mathbf{d}$. Quantification is shown as average basic/acidic ratio \pm s.e.m, ${ }^{* *} P<0.01$ and ${ }^{* *} P<0.001$, compounds versus DMSO (one-way ANOVA, Dunnett's Multiple Comparison Test)

We further analyzed the pharmacological interaction between DCAP and vorinostat in affecting cell viability by Chou-Talalay method for drug combination analysis ${ }^{34}$. Combination Index (CI) plot obtained by dose-response curves with vorinostat, DCAP and a constant ratio combination of vorinostat and DCAP (3:1) showed a synergistic interaction between the two compounds, as indicated by a $\mathrm{CI}<1$ at different Fraction of cell affected (Fa) (Fig. 6b). Further supporting vorinostat/DCAP synergy, CompuSyn generated isobologram showed a marked reduction of drug doses required to achieve a 50 , 75 and $95 \%$ reduction of viable cells $\left(\mathrm{ED}_{50}, \mathrm{ED}_{75}\right.$ and $\mathrm{ED}_{95}$ ) (Fig. 6c).

These data indicate that DCAP significantly improve the anticancer activity of the clinical relevant antineoplastic agent, vorinostat.

\section{Discussion}

A previous screening for novel broad-spectrum antibiotic identified (2-((3-(3,6-dichloro-9H-carbazol-9-yl)-2hydroxypropyl)amino)-2-(hydroxymethyl)propane-1,3-

diol), named DCAP, as a new compound targeting the membranes of both Gram-positive and Gram-negative bacteria $^{27}$. Accordingly, DCAP inhibited the growth of a number of clinical pathogens, including Escherichia coli, Pseudomonas aeruginosa, and Bacillus subtilis ${ }^{27}$.

Here we show that DCAP also possesses autophagymodulating activity. Specifically, DCAP reduced the autophagic flux in a number of human cell lines from diverse tissue sources, as indicated by increased levels of the lipidated LC3 and the autophagy receptor, SQSTM1. The observed accumulation of SQSTM1 and NBR1 in autophagosome-enriched preparations can be attributed to reduced autophagic degradation upon DCAP treatment. CLEM analysis further indicated that DCAPmediated autophagy inhibition derived from a blockade of the last step of the autophagy process. Indeed, DCAPtreated SQSTM1-RFP-expressing cells accumulated numerous autophagolysosome structures enclosing partially degraded material in the corresponding regions with elevated SQSTM1 fluorescent dots. Consistent with this, DCAP inhibited lysosomal acidification without altering lysosomal membrane properties, as indicated by the absence of Galectin 1 punctae accumulation in DCAPtreated cells.

The autophagy inhibitory activity of DCAP therefore suggests its potential use against UTIs, which are among the most frequently recurring infectious diseases in humans ${ }^{35}$. Since Gram-negative UPEC are known to be harbored within autophagosomes, a blockade of autophagy has been proposed as a suitable strategy for reducing UPEC reservoirs ${ }^{18}$. In line with this, UPEC-infected bladder epithelial cells treated with DCAP showed a significant reduction in intracellular bacterial persistence. Furthermore, DCAP treatment did not affect bacterial persistence in epithelial cells lacking the essential autophagy gene, ATG16L1.

The clinically relevant autophagy inhibitor, $\mathrm{CQ}$, has been also shown to reduce intracellular UPEC bacteria, by blocking autophaglysosome maturation ${ }^{10}$. Nonetheless, CQ presents a reduction in neutrophil count (neutropenia) as a common adverse effect ${ }^{36,37}$. Because neutrophils comprise a fundamental protective defense mechanism against pathogens, drug-related neutropenia highly increases the susceptibility to both Gram-positive and Gram-negative bacterial infections ${ }^{38}$. In this context, CQ presents a high liability for its use in antibacterial treatment due to the risk of generating novel bacterial infection. Therefore, compounds with dual inhibitory activity against bacteria and autophagy, such as DCAP, may be a valuable alternative.

Notably, we further showed that DCAP effectively potentiates the anticancer activity of the clinical relevant antineoplastic agent, vorinostat, which similar to $C Q$ presents neutropenia as a common adverse effect ${ }^{39}$. Accordingly, the use of DCAP or DCAP-derived analogs as adjuvant therapy in vorinostat treatment may be a suitable approach to enhance anticancer activity while preventing a chemotherapy-related increased risk of bacterial infections.

Moreover, compared with a combination of single antibacterial and autophagy inhibitory drugs, a molecule having a multiple activity toward autophagy and bacteria would present additional polypharmacology-related 
a

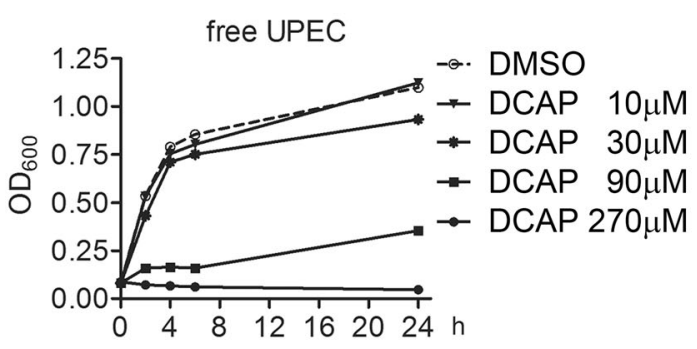

b

\begin{tabular}{cc}
\hline DCAP & $150 \mu \mathrm{M}$ \\
Ampicillin & $70 \mu \mathrm{M}$
\end{tabular}

Chloroquine $>4 \mathrm{mM}$

C

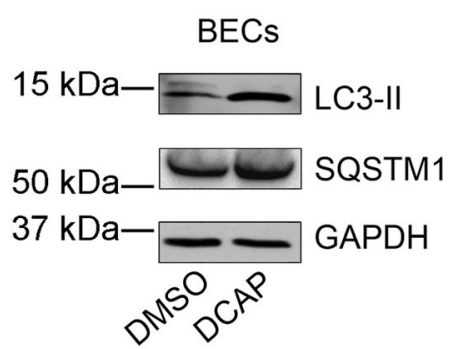

f

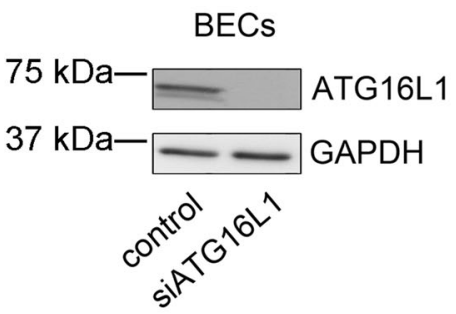

d

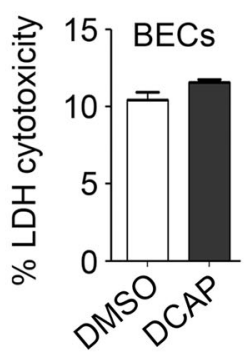

e

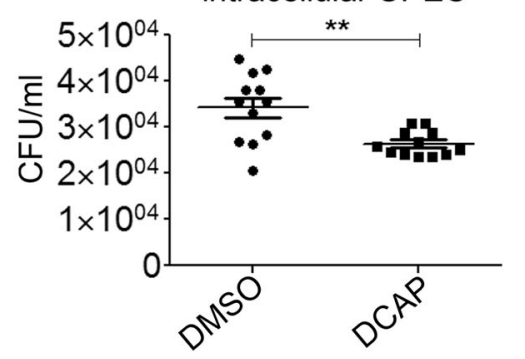

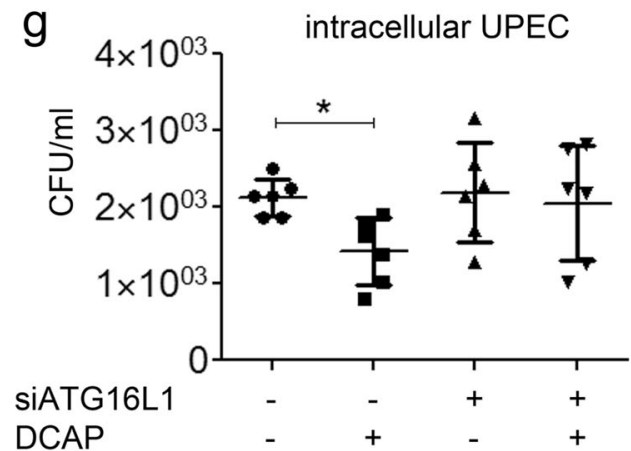

Fig. 5 DCAP reduces autophagy-mediated uropathogenic E.coli infection. a Growth curves of free uropathogenic E. coli bacteria (UPEC) in presence or absence of the indicated DCAP concentrations. $\mathbf{b}$ Measurements of the minimum inhibitory concentration (MIC) of growth against UPEC bacteria of DCAP, CQ or the well-characterized beta-lactam antibiotic, ampicillin. c Immunoblot analysis of protein samples from Bladder Epithelial Cells (BECs) treated $24 \mathrm{~h}$ with vehicle (DMSO) or $10 \mu \mathrm{M}$ DCAP, showing DCAP-mediated accumulation of LC3-II and SQSTM1. GAPDH was used as a loading control. d The potential cytotoxicity of $10 \mu \mathrm{M}$ DCAP against BECs was evaluated by lactate dehydrogenase (LDH) cytotoxic assay. Indicating the tolerability of BECs toward this dose of compound, cells treated for $24 \mathrm{~h}$ showed negligible differences in the release of LDH in the medium, compared with vehicle-treated cells. Reported as percentage of $L D H$ cytotoxicity \pm s.e.m., $n=6$. e BECs were infected with a pathogenic UTI89 E. coli strain. After the removal of extracellular bacteria by gentamycin, an antibiotic that is not permeable through the human cell membrane, BECs were treated with $10 \mu \mathrm{M}$ DCAP or vehicle for $24 \mathrm{~h}$. The counting of intracellular bacteria from DMSO- and DCAP-treated infected cells is reported as an average of the colony-forming unit (CFU)/ml \pm s.e.m., $n=12$. ${ }^{*} P<0.01$ DCAP vs. DMSO (Mann-Whitney test). $\mathbf{f}$ Immunoblot analysis of protein extract from BECs transfected with a siRNA sequences against ATG16L1 or a non-targeting element (control). GAPDH was used as a loading control. g Quantification of intracellular UPEC bacteria in infected siATG16L1 and control BECs treated with $10 \mu \mathrm{M}$ or DMSO. Shown as average CFU/ml \pm s.e. m., $n=7 .{ }^{*} \mathrm{P}<0.05$ DCAP vs. DMSO (Mann-Whitney test)

advantages, including more predictive pharmacokinetics and reduced risk of drug interactions ${ }^{40}$.

Future medicinal chemistry efforts can be now attempted to improve DCAP biological activity toward bacteria and autophagy, and to evaluate/optimize the drug-like properties of this class of compounds. In this context, a preliminary structure activity relationship (SAR) exploration around DCAP has elucidated some chemical and structural features associated with its antibacterial potency ${ }^{41}$. In particular, the DCAP analog,
1-(3,6-dichloro-9H-carbazol-9-yl)-3-[(3-methylbutyl) amino]propan-2-ol (10), inhibited the growth of an E. coli strain at low micromolar doses ${ }^{41}$. Furthermore, analog 10 showed synergistic activity with kanamycin ${ }^{41}$, indicating potential combinatorial use of these antibiotics against UPEC.

The newly identified autophagy inhibition activity of DCAP permits the exploration of whether DCAP analogs with improved antibacterial potency will be still effective in blocking autophagy. Alternatively, these studies will 


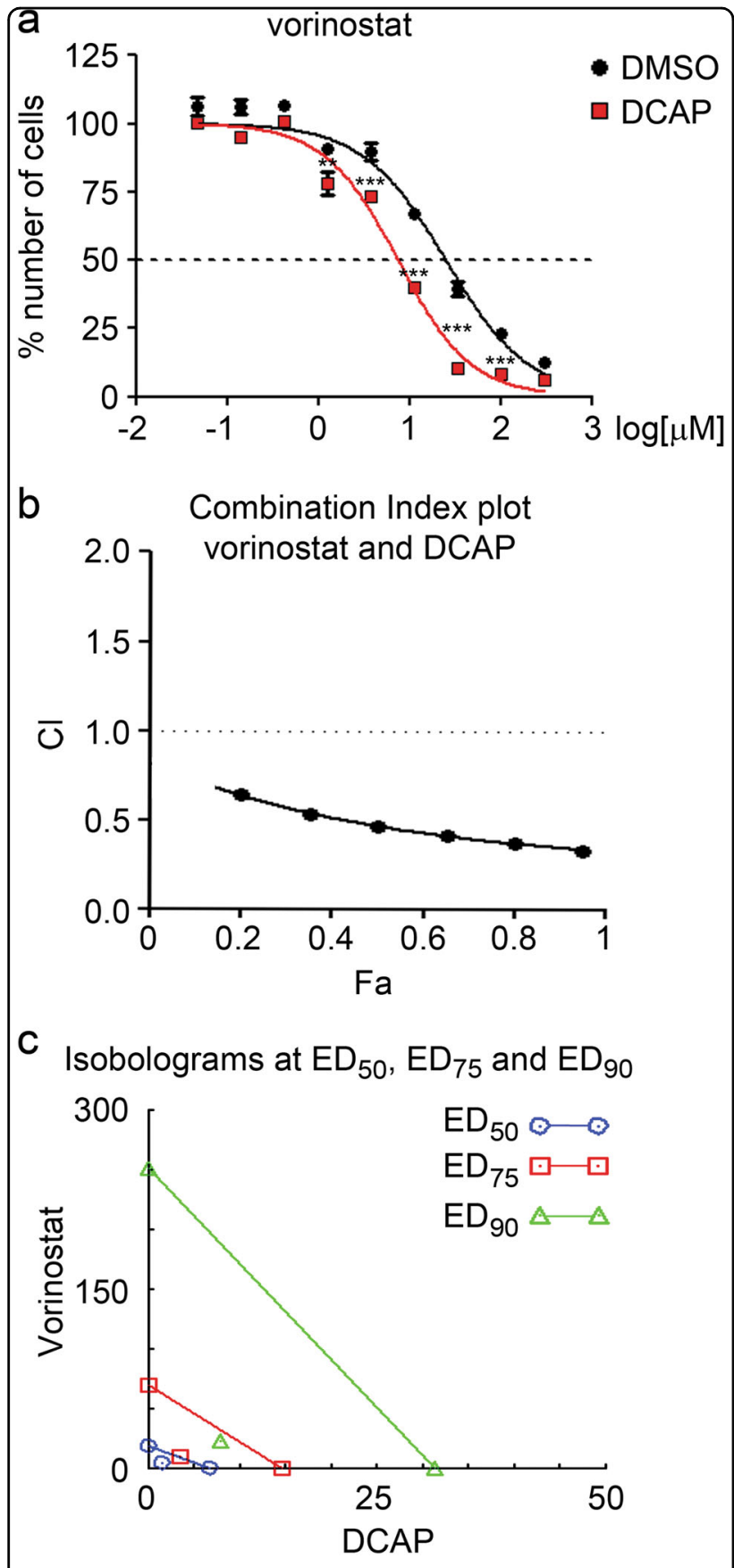

Fig. 6 DCAP improves in vitro anticancer activity of vorinostat. a Concentration response plots of vorinostat cytotoxicity against breast cancer MDA-MB-231 cells in presence (red line) or absence (black line) of $5 \mu \mathrm{M}$ DCAP for $72 \mathrm{~h}$. Values of cells treated with vehicle (DMSO) were set to $100 \%$ of number of cells. Compound concentration is reported as $\log [\mu \mathrm{M}]$. The data expressed as mean $\pm s$. e.m, $n=6 .{ }^{* *} P<0.01$ and ${ }^{* * *} P<0.001$, presence vs. absence of DCAP (two-way ANOVA with Bonferroni post test). $\mathbf{b}$ Combination Index plot obtained from dose-response curves of vorinostat, DCAP and a constant ratio combination of vorinostat and DCAP (3:1) in MDA-MB231 cells. $\mathrm{Fa}=$ Fraction of cells Affected by the treatments. c Isolobograms of drug doses of vorinostat, DCAP or a combination of both compounds, required to achieve a 50\% (blue), 75\% (red) and 95\% (green) reduction of viable cells $\left(\mathrm{ED}_{50}, \mathrm{ED}_{75}\right.$ and $\left.\mathrm{ED}_{95}\right)$ provide important information on the structural and chemical features associated with DCAP-mediated autophagy inhibition, allowing further chemical optimization to improve the bactericidal activity while preserving the autophagy inhibitory feature.

In conclusion, our data identify DCAP as a novel multitarget molecule acting on both autophagy and bacteria, and support the future development of this compound for the delivery of bactericidal autophagy blockers for uropathogenic infections and for antibacterial adjuvant therapy in cancer treatment.

\section{Materials and Methods Chemicals}

2-((3-(3,6-dichloro-9H-carbazol-9-yl)-2-hydroxypropyl) amino)-2-(hydroxymethyl)propane-1,3-diol (DCAP) (Sigma, SML0515-5MG), Carbonyl Cyanide 3-ChloroPhenylhydrazone (CCCP) (Sigma, C2759), H-Leu-Leu-OMe hydrochloride (LLOMe) (Santa Cruz, sc-285992), vorinostat (Sigma, SML0061), chloroquine (Sigma, C6628), bafilomycin A1 (Sigma, B1793), digitonin (Sigma, D141). All compounds were dissolved in dimethyl sulfoxide (DMSO) and a "compound-free" DMSO solution as a comparison (vehicle) was adopted to rule out potential compound-independent biological activity related with the DMSO.

\section{Cell culture}

Human osteosarcoma cells U2OS, human embryonic kidney cells 293 (HEK293), human melanoma cancer cells A375 and human urinary bladder epithelial carcinoma cells (5637, HTB9) were acquired from American Type Culture Collection and the National Collection of Type Cultures (ATCC), were grown in Dulbecco's modified Eagle's medium (DMEM, Euroclone, ECB7501L) with $4.5 \mathrm{~g} / \mathrm{L}$ glucose supplemented with $10 \%$ fetal bovine serum (FBS, Gibco, 10270106), $2 \mathrm{mM} \mathrm{L-glutamine} \mathrm{and} \mathrm{1 \%}$ Penicillin/streptomycin. Breast cancer cells, MDA-MB231 , were cultured in DMEM with $4.5 \mathrm{~g} / \mathrm{L}$ glucose supplemented with 5\% FBS, 4mM L-glutamine and $1 \%$ Penicillin/streptomycin. Bladder carcinoma cells, 5637, and human keratinocyte NCTC2544 were cultured in RPMI 1640 medium (Euroclone, ECB9006L) supplemented with $10 \% \mathrm{FBS}$ and $2 \mathrm{mM} \mathrm{L}$-glutamine. All cell lines were grown at $37^{\circ} \mathrm{C}$ in a humidified atmosphere with $5 \% \mathrm{CO}_{2}$.

\section{Live fluorescence microscopy}

The Premo ${ }^{\text {Th }}$ Autophagy Sensor RFP-p62 Kit and Premo $^{\text {ts }}$ Autophagy Sensor LC3B-RFP (BacMam 2.0) (Invitrogen $^{\text {Tx }}$, P36241 and P36236) were used to overexpress the autophagic marker p62 and LC3 according to company instruction. Briefly, cells were seeded on 48-well plates with bottom glass (Mattek Corporation, P48G- 
1.5-6-F) and the day after infected with viruses expressing p62-RFP or LC3-RFP. Forty-eight hours later, cells were treated and recorded with a fluorescent inverted microscope Eclipse Ti-E (Nikon), with an Okolab incubation system to maintain a humidified atmosphere, $37^{\circ} \mathrm{C}$ and $5 \% \mathrm{CO}_{2}$ during the duration of the experiment.

\section{Immunoblot analysis}

Proteins were extracted by scraping cells in presence of Radio Immunoprecipitation Assay Buffer (RIPA) and protein concentration quantified with BCA assay (Euroclone, EMP014500). Western blot analyses were performed on $20 \mu \mathrm{g}$ of protein extracts, added with $4 \times$ of Laemmli Buffer $+100 \mathrm{mM}$ DTT (Biorad, 161-0747) and denatured at $95^{\circ} \mathrm{C}$ for $5 \mathrm{~min}$. Samples were run on $4-15 \%$ polyacrylamide gels at $90-130 \mathrm{~V}$ and then transferred onto nitrocellulose membrane (GeHealthcare Life Science, 10600001) for $2 \mathrm{~h}$ at $100 \mathrm{~V}$ and $1.5 \mathrm{~A}$ of maximum electric current intensity. After blocking in 5\% non-fat milk in TBS-Tween 20 0,1\% (TBST), membranes were probed overnight at $4{ }^{\circ} \mathrm{C}$ with anti-LC3 primary antibody (1: 2000, MBL), anti-SQSTM1 (1:2000, Santa Cruz), antiNBR1 (1:1000, Cell Signalling), anti-pULK1 s555 (1:1000, Cell Signalling), anti-LAMP1 (1:1000, cell Signalling), anti-GAPDH (1:1000, Invitrogen), anti-Actin (1:100 000, A1978), anti-Tubulin (Cell Signalling, 2128S). Next day, membranes were incubated with horseradish peroxidaseconjugated secondary antibody (goat-anti-rabbit or goatanti-mouse, Millipore) at room temperature for $1 \mathrm{~h}$. Proteins were visualized by the chemiluminescent substrate, ECL Star (Euroclone, EMP001005), using the ImageQuant LAS-4000 Chemiluminescence and Fluorescence Imaging System (Fujitsu Life Science). Densitometry analysis was carried out using ImageJ software 1.51n (Wayne Rasband, National Institutes of Health).

\section{Autophagosome-enriched preparation}

Autophagosome-enriched preparation has been performed as described previously ${ }^{42,43}$. Briefly, cells treated with DCAP or chloroquine (CQ) were collected using trypsin and collected by low speed centrifugation. Packed cell pellets were resuspended in phosphate-buffered saline, PBS, (Sigma-Aldrich, D8537) containing $100 \mu \mathrm{g} / \mathrm{ml}$ of digitonin, then cytosolic and organelle-bound proteins were separated by centrifugation at 7000 r.p.m. for $8 \mathrm{~min}$. Organelle-enriched pellets were solubilized in RIPA buffer, mixed with Laemmli buffer and DTT and boiled as reported above.

\section{Measurement of mitochondrial membrane potential}

Mitochondrial membrane potential was evaluated using the Mitochondrial Membrane Potential Kit (MAK159, Sigma), according to manufacturer instructions. Briefly, cell were seeded in black 96-well culture plates with clear bottom (Perkin Elmer); after $24 \mathrm{~h}$ of treatment with DCAP $10 \mu \mathrm{M}$ or CCCP $20 \mu \mathrm{M}$, cells were stained $1 \mathrm{~h}$ at $37^{\circ} \mathrm{C}$ with JC-10 Dye and then read with Tecan Wallac microplate reader. Ratio $525 \mathrm{~nm} / 590 \mathrm{~nm}$ was used to evaluate membrane potential of treated samples over control.

\section{Lysosomal membrane permeabilization assay}

For lysosomal membrane permeabilization assay, cells were grown on coverslips and treated for $24 \mathrm{~h}$ with $10 \mu \mathrm{M}$ DCAP or $25 \mu \mathrm{M}$ CQ. A treatment with $2 \mathrm{mM}$ LLoME for $2 \mathrm{~h}$ was used as a control ${ }^{32}$. Samples were then fixed with methanol for $10 \mathrm{~min}$, washed twice with PBS, permeabilized with $0.1 \%$ Triton X and blocked with 3\% BSA. Cells staining was performed separately with Galectin 1 antibody (1:1000 in BSA 3\%, Abcam, ab25138) and SQSTM1 (Santa Cruz, B0316) overnight at $4{ }^{\circ} \mathrm{C}$; matching Alexa Fluor 488- or 555-coupled secondary antibodies (Invitrogen, A11029 and A-21429) were probed $1 \mathrm{~h}$ at room temperature with 1:200 dilutions. Images were acquired with $\mathrm{A} 1 \mathrm{R}+\mathrm{l} \mathrm{A} 1+$ confocal laser microscope system Nikon and quantified using ImageJ software $1.51 \mathrm{n}$ (Wayne Rasband, National Institutes of Health).

\section{Lysosomal $\mathrm{pH}$ determination}

The LysoSensor yellow/blue dextran Kit (Invitrogen, L22460) was used to investigate lysosomal acidification alteration. Cells were loaded with $0.5 \mathrm{mg} / \mathrm{ml}$ of dye and treated with DCAP or CQ. After $24 \mathrm{~h}$, cells media were replaced with medium without phenol red and dye. Fluorescence was observed with $\mathrm{A} 1 \mathrm{R}+/ \mathrm{A} 1+$ confocal laser microscope system Nikon. Since the LysoSensor Dye exhibit a dual emission, $\mathrm{pH}$ dependent-fluorescence, ratio of blue over green fluorescence was quantified using ImageJ software 1.51n (Wayne Rasband, National Institutes of Health).

\section{Transmission electron microscopy (TEM) and correlative light electron microscopy (CLEM)}

SQSTM1-RFP expressing cells grown on photoetched gridded coverslips (Bellco Glass Inc., USA) and treated with $10 \mu \mathrm{M}$ DCAP for $24 \mathrm{~h}$. Live cell fluorescence were then imaged using a laser scanning confocal microscope equipped with a resonant scanner (Nikon A1R) and further processed for TEM as previously described ${ }^{7}$. Briefly, the embedded cells were released from the coverslip by transferring the sample between liquid nitrogen and hot water. Sections of about $70 \mathrm{~nm}$ were cut with a Diatome diamond knife on a Leica EM UC6 ultramicrotome. TEM images were obtained with a JEOL JEM 1011 Transmission Electron Microscope operating at $100 \mathrm{kV}$ of acceleration voltage and recorded with a $2 \mathrm{Mp}$ charge-coupled device (CCD) camera (Gatan Orius SC100). Quantifications of degradative vacuoles was performed on more 
than 15 cells/samples on TEM processed samples, as previously described ${ }^{44}$.

\section{In vitro UPEC infections}

In vitro infection assay was performed as described elsewhere ${ }^{10}$. Briefly, a clinical UPEC isolate, UTI89, was grown statically for $17 \mathrm{~h}$ in Luria-Bertani (LB) broth at $37^{\circ}$ $\mathrm{C}$ prior to infection of cells. Confluent 5637 cells were challenged with UPEC. After bacteria were added, plates were centrifuged at $120 \times g$ for $5 \mathrm{~min}$, and then incubated for $1 \mathrm{~h}$ at $37^{\circ} \mathrm{C}$. Extracellular bacteria were then removed by washing twice with PBS, and medium containing 0.1 $\mathrm{mg} / \mathrm{mL}$ gentamicin was added to remove extracellular bacteria. 5637 cells were then incubated in gentamicincontaining medium in presence of DCAP for an additional $23 \mathrm{~h}$ (referred to as $24 \mathrm{~h}$ post infection [hpi]). Next day, 5637 cells were washed and treated with $0.1 \%$ TritonX 100 to release bacteria. Serial dilutions of bacteria were plated on LB agar, and colony-forming units were counted.

\section{Cytotoxicity assay}

Cell counting was performed with Countess II Automated Cell Counter (Thermofisher) in presence of Trypan blue dye to discriminate live and death cells. Proliferation and viability was assessed by Cyquant assay (Invitrogen) as previously described ${ }^{7}$. For drug interaction analysis, combination Index $(\mathrm{CI})$ plot and isobologram were generated by Compusyn software ${ }^{34}$.

\section{Statistical analysis}

Log(inhibitor)-versus-response curves and statistical analysis tests were conducted with GraphPad Prism software (San Diego, CA, USA). A $p$ value $<0.05$ was considered significant.

\section{Acknowledgements}

This work was supported in part by the National Institutes of Health, grant numbers R01 HD091218 and R01 DK100644 (to IUM), and by the Associazione Italiana per la Ricerca sul Cancro (AIRC), grant number IG 17005 (to BG). We thank the Nikon Imaging Centre of Istituto Italiano di Tecnologia for its excellent assistance on fluorescence microscopy.

\section{Author details \\ 'Laboratory of Molecular Medicine, Fondazione Istituto Italiano di Tecnologia, via Morego 30, 16163 Genova, Italy. ${ }^{2}$ Electron Microscopy facility, Fondazione Istituto Italiano di Tecnologia, via Morego 30, 16163 Genova, Italy. ${ }^{3}$ Department of Obstetrics \& Gynecology, Washington University School of Medicine, St. Louis, MO 63110, USA. ${ }^{4}$ Centre for Reproductive Health Sciences, Washington University School of Medicine, St. Louis, MO 63110, USA}

\section{Conflict of interest}

The authors declare that they have no conflict of interest.

\section{Publisher's note}

Springer Nature remains neutral with regard to jurisdictional claims in published maps and institutional affiliations.
Supplementary Information accompanies this paper at (https://doi.org/ 10.1038/s41419-018-0786-4).

Received: 19 January 2018 Revised: 8 June 2018 Accepted: 12 June 2018 Published online: 13 July 2018

\section{References}

1. Varshavsky, A. The Ubiquitin system, autophagy, and regulated protein degradation. Annu. Rev. Biochem. 86, 123-128 (2017).

2. Galluzzi, L. et al. Molecular definitions of autophagy and related processes. EMBO J. 36, e201796697 (2017).

3. Hurley, J. H. \& Young, L. N. Mechanisms of autophagy initiation. Annu. Rev. Biochem. 86, 225-244 (2017).

4. Suzuki, H., Osawa, T., Fujioka, Y. \& Noda, N. N. Structural biology of the core autophagy machinery. Curr. Opin. Struct. Biol. 43, 10-17 (2017).

5. Thelen, A. M. \& Zoncu, R. Emerging roles for the lysosome in lipid metabolism. Trends Cell Biol. 27, 833-850 (2017).

6. Bauckman, K. A., Owusu-Boaitey, N. \& Mysorekar, I. U. Selective autophagy: xenophagy. Methods 75, 120-127 (2015).

7. De Mei, C. et al. Dual inhibition of REV-ERB $\beta$ and autophagy as a novel pharmacological approach to induce cytotoxicity in cancer cells. Oncogene $\mathbf{3 4}$ 2597-2608 (2015)

8. Pankiv, S. et al. p62/SOSTM1 binds directly to Atg8/LC3 to facilitate degradation of ubiquitinated protein aggregates by autophagy. J. Biol. Chem. 282, 24131-24145 (2007).

9. Klionsky, D. J. et al. Guidelines for the use and interpretation of assays for monitoring autophagy. Autophagy 12, 1-222 (2016).

10. Bauckman, K. A. \& Mysorekar, I. U. Ferritinophagy drives uropathogenic Escherichia coli persistence in bladder epithelial cells. Autophagy 12, 850-863 (2016).

11. Kroemer, G., Marino, G. \& Levine, B. Autophagy and the integrated stress response. Mol. Cell 40, 280-293 (2010).

12. Murrow, L. \& Debnath, J. Autophagy as a stress-response and quality-contro mechanism: implications for cell injury and human disease. Annu. Rev. Pathol. 8, 105-137 (2013).

13. Patel, K. K. \& Stappenbeck, T. S. Autophagy and intestinal homeostasis. Annu. Rev. Physiol. 75, 241-262 (2013).

14. Rodolfo, C., Di Bartolomeo, S. \& Cecconi, F. Autophagy in stem and progenitor cells. Cell. Mol. Life Sci. 73, 475-496 (2016).

15. Sepe, S. et al. Expression of Ambra1 in mouse brain during physiological and Alzheimer type aging. Neurobiol. Aging 35, 96-108 (2014).

16. Mizushima, N. \& Levine, B. Autophagy in mammalian development and differentiation. Nat. Cell Biol. 12, 823-830 (2010).

17. Tong, D. \& Hill, J. A. Spermidine promotes cardioprotective autophagy. Circ. Res. 120, 1229-1231 (2017).

18. Wang, C. et al. Atg16L1 deficiency confers protection from uropathogenic Escherichia coli infection in vivo. Proc. Natl Acad. Sci. 109, 11008-11013 (2012).

19. Wang, C., Symington, J. W. \& Mysorekar, I. U. ATG16L1 and pathogenesis of urinary tract infections. Autophagy 8, 1693-1694 (2012).

20. Jiang, X., Overholtzer, M. \& Thompson, C. B. Autophagy in cellular metabolism and cancer. J. Clin. Invest. 125, 47-54 (2015).

21. Levy, J. M. M., Towers, C. G. \& Thorburn, A. Targeting autophagy in cancer. Nat. Rev. Cancer 17, 528-542 (2017)

22. Santana-Codina, N., Mancias, J. D. \& Kimmelman, A. C. The role of autophagy in cancer. Annu. Rev. Cancer Biol. 1, 19-39 (2017).

23. Carew, J. S. et al. Lucanthone is a novel inhibitor of autophagy that induces cathepsin D-mediated apoptosis. J. Biol. Chem. 286, 6602-6613 (2011).

24. Pietrocola, F. et al. Autophagy induction for the treatment of cancer. Autophagy 12, 1962-1964 (2016).

25. McAfee, Q. et al. Autophagy inhibitor Lys05 has single-agent antitumor activity and reproduces the phenotype of a genetic autophagy deficiency. Proc. Natl Acad. Sci. 109, 8253-8258 (2012).

26. Torrente, E. et al. Synthesis and in vitro anticancer activity of the first class of dual inhibitors of REV-ERBß and autophagy. J. Med. Chem. 58, 5900-5915 (2015).

27. Heinrich, V., Hurley, K, Santos, T. \& Weibel, D. DCAP: a broad-spectrum antibiotic that targets the cytoplasmic membrane of bacteria. FASEEB J. 29, 575-576 (2015). 
28. Kwon, K.-Y., Viollet, B. \& Yoo, O. J. CCCP induces autophagy in an AMPKindependent manner. Biochem. Biophys. Res. Commun. 416, 343-348 (2011).

29. Kirkin, $V$. et al. A role for NBR1 in autophagosomal degradation of ubiquitinated substrates. Mol. Cell 33, 505-516 (2009).

30. Pietrocola, F. et al. Spermidine induces autophagy by inhibiting the acetyltransferase EP300. Cell Death \& Differ. 22, 509-516 (2015).

31. Boya, P. \& Kroemer, G. Lysosomal membrane permeabilization in cell death. Oncogene 27, 6434-6451 (2008).

32. Aits, S. et al. Sensitive detection of lysosomal membrane permeabilization by lysosomal galectin puncta assay. Autophagy 11, 1408-1424 (2015).

33. Kroemer, G. Autophagy: a druggable process that is deregulated in aging and human disease. J. Clin. Invest. 125, 1-4 (2015).

34. Chou, T.-C. Drug combination studies and their synergy quantification using the Chou-Talalay method. Cancer Res. 70, 440-446 (2010).

35. Dielubanza, E. J. \& Schaeffer, A. J. Urinary tract infections in women. Med. Clin. North Am. 95, 27-41 (2011).

36. Curtis, B. Drug-induced immune neutropenia/agranulocytosis. Immunohematology 30, 95-101 (2014).

37. Rongey, C. \& Cherian, S. Effects of chloroquine therapy on white blood cells. Blood 126, 1149-1149 (2015)
38. Nesher, L. \& Rolston, K. V. I. The current spectrum of infection in cancer patients with chemotherapy related neutropenia. Infection 42, 5-13 (2014).

39. Kepp, O., Galluzzi, L. \& Kroemer, G. Immune effectors required for the ther apeutic activity of vorinostat. Oncoimmunology 2, e27157 (2013).

40. Ramsay, R. R., Popovic-Nikolic, M. R., Nikolic, K., Uliassi, E. \& Bolognesi, M. L. A perspective on multi-target drug discovery and design for complex diseases. Clin. Transl. Med. 7, 3 (2018).

41. Hurley, K. A., Heinrich, V. A., Hershfield, J. R., Demons, S. T. \& Weibel, D. B. Membrane-targeting DCAP analogues with broad-spectrum antibiotic activity against pathogenic bacteria. ACS Med. Chem. Lett. 6, 466-471 (2015).

42. Kaminskyy, V., Abdi, A. \& Zhivotovsky, B. A quantitative assay for the monitoring of autophagosome accumulation in different phases of the cell cycle. Autophagy 7, 83-90 (2011).

43. Allavena, G. et al. Suppressed translation as a mechanism of initiation of CASP8 (caspase 8)-dependent apoptosis in autophagy-deficient NSCLC cells under nutrient limitation. Autophagy. 14, 252-268 (2017).

44. Parodi, C. et al. Autophagy is essential for maintaining the growth of a human (mini-) organ: evidence from scalp hair follicle organ culture. PLoS Biol. 16 e2002864 (2018). 